\section{Brain-computer interface using a simplified functional near-infrared spectroscopy system}

To cite this article: Shirley M Coyle et al 2007 J. Neural Eng. 4219

View the article online for updates and enhancements.
You may also like

- Decoding human mental states by whole-
head EEG+fNIRS during category fluency
task performance
Ahmet Omurtag, Haleh Aghajani and
Hasan Onur Keles
- Imagined speech increases the
hemodynamic response and functional
Connectivity of the dorsal motor cortex
al.
- A hyopeng Si, Sicheng Li, Shaoxin Xiang et
signals improves the performance of
decoding motor imagery of both force and
speed of hand clenching
Xuxian Yin, Baolei Xu, Changhao Jiang et
al.




\title{
Brain-computer interface using a simplified functional near-infrared spectroscopy system
}

\author{
Shirley M Coyle ${ }^{1}$, Tomás E Ward ${ }^{2}$ and Charles M Markham ${ }^{3}$ \\ ${ }^{1}$ National Centre for Sensor Research, Dublin City University, Glasnevin, Dublin 9, Ireland \\ ${ }^{2}$ Department of Electronic Engineering, National University of Ireland Maynooth, Maynooth, \\ County Kildare, Ireland \\ ${ }^{3}$ Department of Computer Science, National University of Ireland Maynooth, Maynooth, \\ County Kildare, Ireland \\ E-mail: shirley.coyle@dcu.ie
}

Received 24 October 2006

Accepted for publication 4 April 2007

Published 27 April 2007

Online at stacks.iop.org/JNE/4/219

\begin{abstract}
A brain-computer interface (BCI) is a device that allows a user to communicate with external devices through thought processes alone. A novel signal acquisition tool for BCIs is near-infrared spectroscopy (NIRS), an optical technique to measure localized cortical brain activity. The benefits of using this non-invasive modality are safety, portability and accessibility. A number of commercial multi-channel NIRS system are available; however we have developed a straightforward custom-built system to investigate the functionality of a fNIRS-BCI system. This work describes the construction of the device, the principles of operation and the implementation of a fNIRS-BCI application, 'Mindswitch' that harnesses motor imagery for control. Analysis is performed online and feedback of performance is presented to the user. Mindswitch presents a basic 'on/off' switching option to the user, where selection of either state takes $1 \mathrm{~min}$. Initial results show that fNIRS can support simple BCI functionality and shows much potential. Although performance may be currently inferior to many EEG systems, there is much scope for development particularly with more sophisticated signal processing and classification techniques. We hope that by presenting fNIRS as an accessible and affordable option, a new avenue of exploration will open within the BCI research community and stimulate further research in fNIRS-BCIs.
\end{abstract}

(Some figures in this article are in colour only in the electronic version)

\section{Introduction}

This paper describes principles, experiments and progress in harnessing simplified near-infrared spectroscopic systems as non-invasive instrumentation for brain-computer interfaces (BCIs). A BCI is a device that translates physiological measures of volitional thought processes into control signals capable of driving external computers, independent of the peripheral nervous system. Speed, accuracy, ease of use and length of training period are key criteria for assessing BCI innovations. Currently, no existing system performs well under all these headings [1] and consequently there is concerted research in developing new and alternative technologies for practical systems.

Near-infrared spectroscopy (NIRS) is a relatively new measurement modality that offers a portable, low-cost, sensitive option for BCI development. While still in its infancy in terms of BCI research, NIRS-derived optical responses have been demonstrated in a number of cognitive and motor task studies [2-4]. Such mental tasks are particularly suitable for BCI development. At present the measurement of electrical activity from the brain, using electroencephalography (EEG) or electrocorticography $(\mathrm{ECoG})$, is the favoured method for harnessing such tasks for BCI development [1, 5-8]. 
Other functional imaging methods including fMRI and MEG have been demonstrated, but are relatively unsuitable due to the impracticalities of the equipment $[9,10]$. Invasive procedures involving PET and SPECT are undesirable and not suitable for long-term use due to long time constants and the exposure to radioactive tracers. The use of functional near-infrared spectroscopy as demonstrated here offers an alternative investigation route. It is a safe, inexpensive and practical option for BCI signal acquisition, allowing for direct measurement of localized cognitive activity with minimal training. Its main drawback is the speed of operation which is limited due to the nature of the metabolic response responsible for the signal. Another important issue, as with most physiological monitoring, is ensuring good coupling when connecting the sensors to the body. A careful design of the optodes and coupling mechanisms is a critical factor as motion and obstruction of hair can severely affect performance and signal quality.

The principal contribution of this paper is the detailed exposition of a single site simplified real-time functional (NIRS) system as an instrument for BCI development. The suitability of our approach is demonstrated through the assessment of NIRS signal characteristics and detectable physiological effects that are suitable for BCI development $[11,12]$. The system operates in a synchronous mode, i.e. the user is prompted for their input during defined time intervals. The results of preliminary trials are presented using a straightforward classification method for online analysis.

\section{Methods}

\section{NIRS_the optical signal}

An optical window exists (approximately 650-900 nm) where light is able to penetrate the outer tissues of the human head, including the cranium and the various meninges and fluids surrounding the brain. Light entering the head at a particular point becomes diffuse as the photons undergo multiple scattering events. A number of photons are absorbed while others continue to be scattered, following a random walk path movement through the medium. Some photons may be back reflected from tissue and exit the surface up to several centimetres from the original point source location. The amount of back-reflected photons depends on the optical properties of the tissue and may be detected using an appropriate optical source and a suitably placed photon detector. Both experimental and theoretical results suggest that photons collected carry information on optical properties and their changes at depths of up to approximately $2 \mathrm{~cm}$ in the adult human head [13], reaching the cortical surface. Changes in tissue oxygenation associated with brain activity modulate the absorption and scattering of these near-infrared (NIR) photons and hence affect the detected light levels emerging from the tissue (1-2\% changes in signal amplitude) [14]. Measurement of light attenuation resulting from photon interactions is the basic principle underlying our approach. The attenuation due to absorption and scattering effects may be described by a modified version of the Beer-Lambert law:

$$
A=\log _{10} \frac{I_{\mathrm{o}}}{I}=\alpha c L B+G,
$$

where $A$ is attenuation, $I_{\mathrm{o}}$ is the incident light intensity $(\mathrm{mW})$, $I$ is the transmitted light intensity $(\mathrm{mW}), \alpha$ is the specific extinction coefficient $\left(\mathrm{mol}^{-1} \mathrm{~m}^{-1}\right), c$ is the concentration of the absorber (mol), $L$ is the distance between the source and the detector (m), $B$ is the differential pathlength factor and $G$ is a term to account for scattering losses.

The algorithm implemented by the optical BCI to calculate changes in haemoglobin levels is based on an algorithm described by Cope and Delpy [15] that relies on the modified Beer-Lambert law

$$
\Delta A=\left(\alpha_{\mathrm{HbO}} \Delta c_{\mathrm{HbO}}+\alpha_{\mathrm{Hb}} \Delta c_{\mathrm{Hb}}\right) \mathrm{BL},
$$

where $\Delta A$ is the change in light attenuation, $\alpha_{\mathrm{HbO}}$ and $\alpha_{\mathrm{Hb}}$ are the specific extinction coefficients of oxy-haemoglobin $(\mathrm{HbO})$ and deoxy-haemoglobin $(\mathrm{Hb})$, respectively, and $\Delta c_{\mathrm{HbO}}$ and $\Delta c_{\mathrm{Hb}}$ are the concentration changes in $\mathrm{HbO}$ and $\mathrm{Hb}$, respectively.

The algorithm can be applied to multiple wavelengths of light; in this case, two are used. The two NIR light sources, at $760 \mathrm{~nm}$ and $880 \mathrm{~nm}$, are chosen to be on either side of the isobestic point to evaluate the two chromophores of interest, i.e. $\mathrm{Hb}$ and $\mathrm{HbO}$.

Various fNIRS imaging studies have reported optical responses due to motor, cognitive, visual and auditory functions [16], and in a previous study we reported an optical response due to motor imagery related to localized cerebral blood flow changes in the sensorimotor cortex [11]. Analysis of the signals showed characteristic changes in the deoxygenated and oxygenated haemoglobin concentrations during mental activation. Typically, an increase in oxyhaemoglobin ( $\mathrm{HbO}$ ) and a decrease in deoxy-haemoglobin $(\mathrm{Hb})$ occur after the onset of movement execution or imagery. From our preliminary studies $\mathrm{HbO}$ concentration was found to exhibit a greater, more significant change during mental tasks. The optical BCI detects and characterizes changes in $\mathrm{HbO}$ in real time, giving user feedback, which could also be translated to control external devices.

\section{Functional NIRS measurement and instrumentation}

Functional NIRS measurements are made using one of the three methods, namely continuous wave $(\mathrm{CW})$, time-resolved (TR) and frequency domain (FD). Each of these manifests specific strengths and weaknesses when considered in the light of a particular application. Broadly, time-resolved and frequency domain methods yield quantitative chromophore concentration measurements at the cost of increased apparatus complexity while CW methods in their most basic guise yield only qualitative information on such changes. This is because a direct measurement of photon pathlengths cannot be made, and therefore the differential pathlength factor must be estimated based on empirical studies. Time-resolved spectroscopy can gauge the pathlength by measuring the time taken for photons to traverse a medium, which is termed 'time of flight'. In the frequency domain approach, a light source is modulated at a very high frequency (hundreds of megahertz) and the light migrating through tissue undergoes both amplitude and phase shifts. The phase delay is related to the average time of flight of the photons, and the differential pathlength can be 


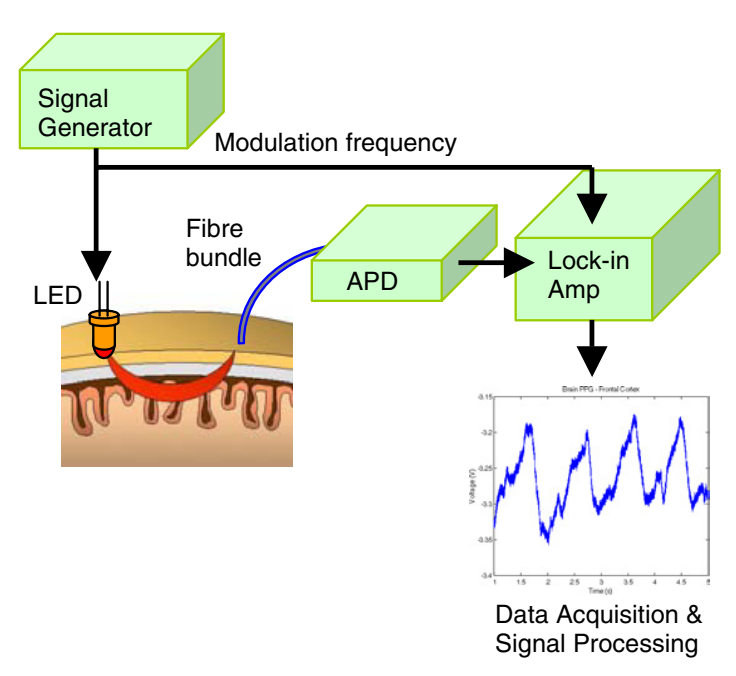

Figure 1. Components of a continuous wave NIR system.

calculated. Using CW a light source is switched on constantly, or modulated at low frequencies of a few kilohertz. Magnitude changes in the detected light signal are used to evaluate tissue attenuation. This can be implemented easily on account of its simplicity and flexibility and yields an inexpensive practical system. Further information on NIRS techniques, including an excellent introduction to NIRS instrumentation, is given by Rolfe [13]. As qualitative measurements are sufficient for detecting haemodynamics changes, the $\mathrm{CW}$ method was chosen for implementation in this BCI application.

\section{Functional NIRS-BCI system design}

The fundamental components of any fNIRS system are the light source and the detector although adequate care must also be taken in optode design, driving electronics, data acquisition and signal processing. The fNIRS system we have built for this study is shown in figure 1. Light emitting diodes (LEDs) at wavelengths of $760 \mathrm{~nm}$ and $880 \mathrm{~nm}$ are used (Opto-Diode Inc. APT-0010/OD-880F), each having a narrow beam angle of $8^{\circ}$ and a spectral bandwidth at $50 \%$ of $30 \mathrm{~nm}$ and $80 \mathrm{~nm}$ respectively. These two wavelengths are chosen to allow blood oxygenation to be measured, as haemoglobin has different attenuation spectra depending on its oxygenation state. The LEDs emit a combined average power of less than $2 \mathrm{~mW} \mathrm{~mm}^{-2}$ and are modulated in the low kilohertz range. Given their small dimensions, the LEDs may be placed in direct contact with the scalp. The detector is an avalanche photodiode (APD), Hamamatsu C5460-01, which is connected 3-4 cm from the light source via a $3 \mathrm{~mm}$ diameter, $1 \mathrm{~m}$ long, fibre optic bundle. Lock-in amplifiers (Signal Recovery Model 7265) are used to identify each light source, while also reducing noise due to ambient light. The data are recorded at a sampling rate of $100 \mathrm{~Hz}$ by a 16 bit A/D data acquisition card, Keithley PCMCIA16AI.

\section{Optode design and placement}

A careful design must be applied to the mechanics of coupling light from the optical sources and detectors (optodes) to and from the subject's head. It is important to maintain a good connection with the scalp and also to push hair aside. Dark hair obstructing the photon's path can significantly attenuate the signal, and every effort must be made to overcome this problem; combing hair out of the way, fixing with hairgel and hair clips are an imperative part of the experimental regime. Although the fundamental method of connecting optodes to a subject's head may seem trivial, it seems to have the greatest bearing on the system performance as it has a great influence on the signal quality. A stable connection between the optodes and scalp is vital to ensure that the pathlength of the photons does not change and also to prevent ambient light saturating the detector. Such effects create spurious motion artefacts affecting the validity of the detected NIR signals. The challenge is to ensure rigid optode positioning while still allowing for subject comfort. Solutions to date include modified cycle helmets, thermoplastic moulded to the contours of each subject's head, spring-loaded fibres attached to semirigid plastic forms and fibres embedded in rubber forms [17]. Here an alternative approach was taken using a mechanical mounting structure to support the weight of the optodes while also cradling the subject's head, avoiding the effects of strain and discomfort that can occur with tight restrictive straps. The mechanical mounting system allows the person to sit comfortably, while a supporting structure is positioned about the person to hold the optodes in place. Mechanical supports for the optodes are connected to a seat with an adjustable back incline. This provides the option of conducting studies where the subject is supine, reducing certain physiological noise effects such as the Mayer wave [18]. This was shown for measurements taken from the same subject assuming different postural positions [3]. The mechanical framework is shown in figure 2(a). The position of the supporting structure can be altered by varying the height and also within the horizontal plane to allow for varying spinal curvatures, i.e. a subject's head sits more forward if there is a large curvature of his back. The framework allows optodes to be positioned over areas of the head that are generally of interest in functional brain imaging studies, e.g., visual and motor association areas. The physical framework consists of semicircular rails onto which optode holders can be attached and removed. The optode holders can slide along these rails and be secured into a required position. One of the semicircular rails curves above the left and right sensorimotor cortices, which provides good optode placement for investigating motor imagery [11].

In terms of optode placement, there is currently no standardized placement scheme for NIRS measurements. In order to be able to reproduce measurements from the same subjects and also to ensure consistency between studies, a welldefined method of performing physical measurements needs to be defined. This is especially important in the absence of fMRI information, where anatomical and functional details are available. As optical measurements are typically taken based on the measurements of the EEG 10-20 system we have proposed a type of polar coordinate system in terms of the 10-20 positions, where the optode position is described in terms of distance and angle with respect to a defined EEG $10-20$ position. The nomenclature is as follows ' $10-20$ position origin; distance from origin (angle wrt Cz)'. The origin is based 


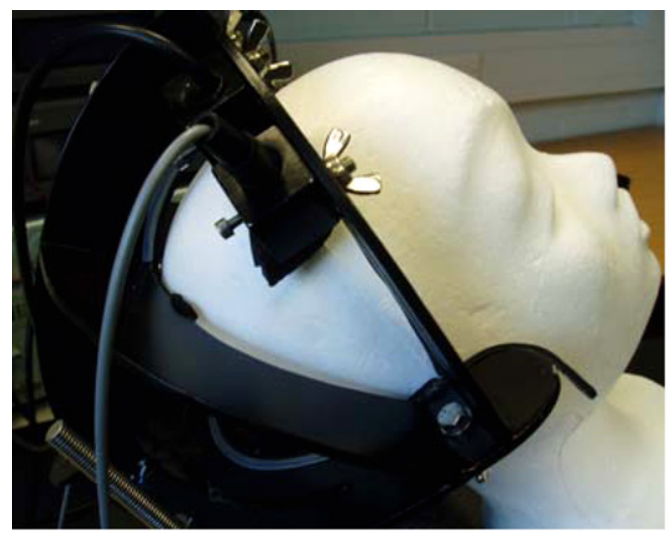

(a)

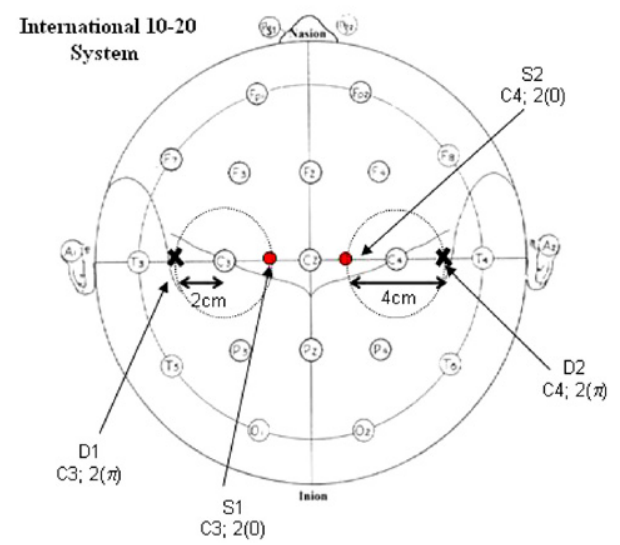

(b)

Figure 2. (a) Mechanical mounting system and (b) proposed optode placement system based on the conventional EEG 10-20 system.

on the closest $10-20$ position and the vertex, i.e. $\mathrm{Cz}$, is used as a general reference point to describe the placement angle. The positions of a source (S1) and detector (D1) placed $4 \mathrm{~cm}$ apart, lying $2 \mathrm{~cm}$ directly above and below $\mathrm{C} 3$ respectively, can be described by $\mathrm{C} 3 ; 2(0)$ and $\mathrm{C} 3 ; 2(\pi)$, as shown in figure 2(b). Similarly for optodes placed around $\mathrm{C} 4$, source (S2) and detector (D2) are placed at C4; 2(0) and C4; $2(\pi)$. Such a standardized placement scheme is important for concurrent measurements with other measurement technologies such as EEG.

\section{Software}

We have previously described the ability of our NIRS system to detect brain vascular dynamical changes associated with motor imagery [19]. Further work involving a number of preliminary brain-computer interface applications is described elsewhere [3]. Here, in the interests of brevity we choose to describe the experimental application which best epitomizes the principles underlying our fNIRS-based BCI. The simplest BCI requires a binary (yes/no) signal, which is achievable using a singlechannel system. The optical response that is detected at the left or right motor cortex, due to imagined hand movement, may be used to provide this binary channel.

The goal of this experiment was to demonstrate a binary switching action controlled using mental imagery taskswe refer to this paradigm as a 'Mindswitch'. The system operates in a synchronous mode, i.e. the system is active only during defined periods [20]. In this model, two options are presented to the user and highlighted in turn by the controlling software. When the user's desired target is highlighted, he/she performs imagery tasks to indicate his/her selection. The device monitors motor cortex activity during both options and then compares the response to each. The option associated with a larger change in $\mathrm{HbO}$ is used to assess the physiological response. The $\mathrm{HbO}$ signal was chosen for classification as this was noted to exhibit larger signal changes than $\mathrm{Hb}[3,11]$.

\section{Experimental procedure}

Three healthy subjects (two right-handed and one left-handed, average age 35) participated in the evaluation of 'Mindswitch'.
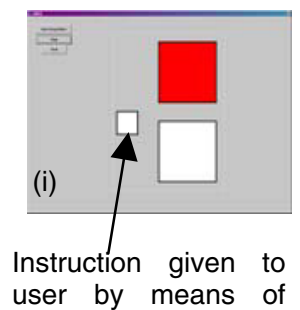
small square (white or red)
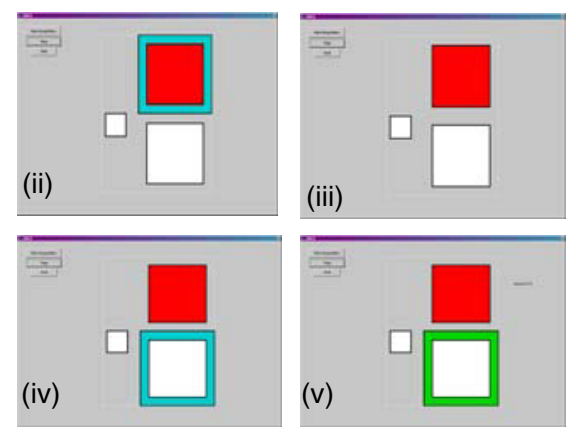

Figure 3. (i)-(v) Sequence of operations in Mindswitch.

Motor imagery was used as the mental strategy for controlling the application. Subjects resided comfortably in a supine position throughout the experimental trials and optodes were placed above the motor cortex at $\mathrm{C} 3 ; 2(0)$ and $\mathrm{C} 3 ; 2(\pi)$ for the right-handed subjects and $\mathrm{C} 4 ; 2(0)$ and $\mathrm{C} 4 ; 2(\pi)$ for the left-handed subjects. Subjects were instructed to imagine softly clenching a ball with their right hand, while their arms rested relaxed on the armrest. They were asked to imagine the kinaesthetic experience of movement while avoiding muscle tension [21].

Subject 2 and subject 3 each participated in one experimental session. To investigate performance over time, subject 1 participated in four experimental sessions spread over a week. Subjects 1 and 2 had participated previously in motor imagery experiments, while subject 3 received no prior training. Each session consisted of two experimental runs, with a break in between. During each experimental run, the subjects were asked to select a target ten times. Two target squares are presented to the user for selection-a red square and a white square. The sequence of procedures involved in choosing a target is shown in figure 3. Selection of a target takes a total duration of $1 \mathrm{~min}$, and involves the following procedure.

(1) The first $15 \mathrm{~s}$ is a resting period, establishing a baseline condition. In the last $5 \mathrm{~s}$ of this period, a command is given to the user by means of a small coloured square indicating which target must be selected. 


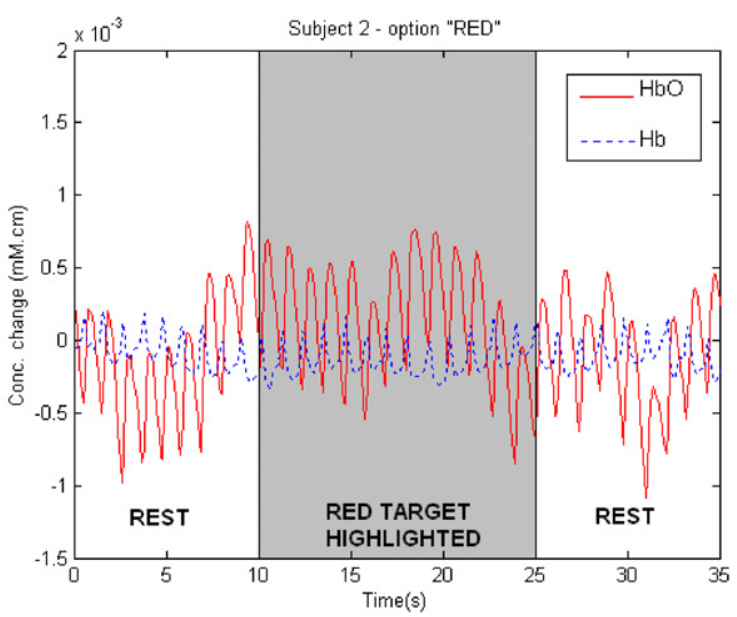

(a)

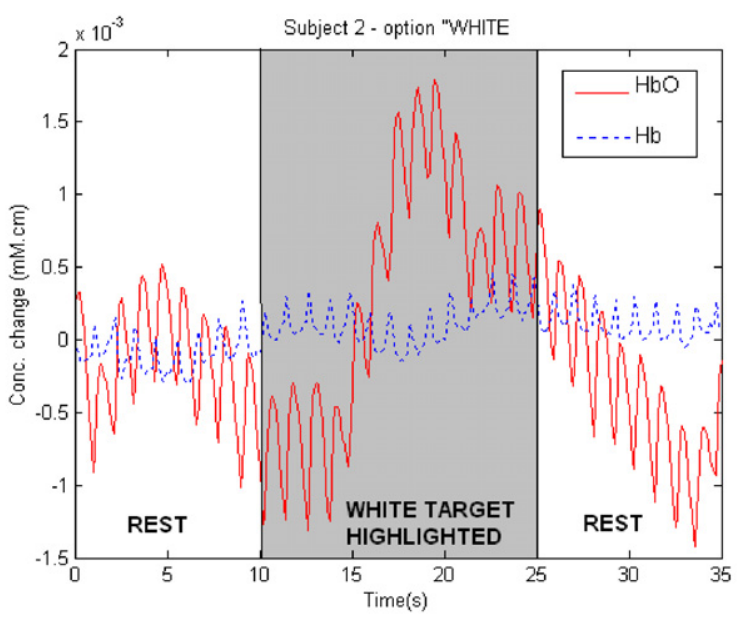

(b)

Figure 4. $\mathrm{HbO} / \mathrm{Hb}$ response during a single trial (raw data): (a) when the red square option is highlighted, (b) when the white square option is highlighted. The average $\mathrm{HbO}$ signal is evaluated during each time interval and average $\mathrm{HbO}$ changes are evaluated for each option and compared. In this trial, the white option is selected.

(2) In the next $15 \mathrm{~s}$ the upper target, the red square, is highlighted. If the user wishes to select the highlighted target, he/she must perform imagery tasks during this time.

(3) The following $15 \mathrm{~s}$ is another rest state allowing the $\mathrm{HbO}$ signal to return to baseline if necessary.

(4) During the next $15 \mathrm{~s}$ the lower target is highlighted, and again the user may perform imagery if the highlighted target is desired.

(5) Rest follows, with the decision being presented to the user and the score updated (number of correct trials/total number of trials).

\section{Data processing and classification}

Signal processing and user feedback are performed online by the BCI. HbO concentration changes are evaluated from the detected light signal, based on the algorithm described by Cope [15]. The average $\mathrm{HbO}$ signal is evaluated during the last $10 \mathrm{~s}$ of the rest period and during the last $10 \mathrm{~s}$ of the target selection period. In this way, for each trial, the average $\mathrm{HbO}$ change is established for the red target and for the white target. A decision is made by the system based on which target elicited a larger $\mathrm{HbO}$ signal change. Figures 4(a) and (b) show the haemodynamic response for a single trial, and illustrate the time intervals used to evaluate HbO signal changes. The signals presented here are raw data. The oscillatory component is due to the cardiac cycle, to give an impression of the magnitude of the functional haemodynamic response. From these data it is clear that even before signal processing, a significant response can be observed by eye (only the algorithm to calculate $\mathrm{Hb} / \mathrm{HbO}$ has been applied to the detected light signals). The $\mathrm{Hb}$ signal, also shown in figure 4, is not currently considered for classification by the BCI but we intend to investigate this response further. From these graphs, it is clear that the white square option causes a larger change in the $\mathrm{HbO}$ signal and is therefore selected. The decision is displayed to the user at the end of

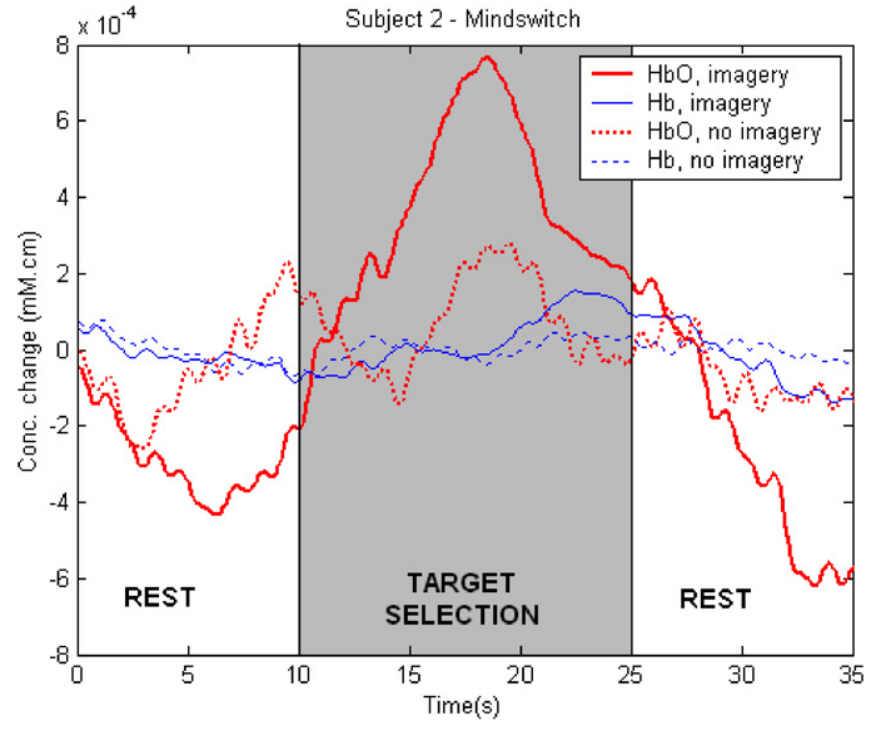

Figure 5. Subject 2-haemodynamic response measured at the right motor cortex during target selection trials. Signal has been low-pass filtered to reduce pulse artefact. Average response is shown for ten trials, i.e. one experimental run. The thicker line is the average of the ten imagery tasks and the thinner line the average of the ten epochs when no imagery was performed.

each trial and a score out of ten is updated on the visual display throughout the trials.

\section{Results}

Results are presented based on 60 trials for subject 1,10 trials for subject 2 and 20 trials for subject 3. One session for subject 1 was rejected as the subject reported difficulty in performing imagery due to general fatigue, similarly for one experimental run for subject 2. The typical response for subject 2 for an experimental run of ten trials is shown in figure 5 . The average response during ten motor imagery tasks is shown versus the average response during ten epochs of no 
Table 1. Average HbO signal changes during fNIRS-BCI trials. Each experimental run consists of ten trials; there are four possible outcomes: (i) red square is targeted and selected (correct response), (ii) red square is targeted but white is selected (incorrect response), (iii) white is targeted and selected (correct response), (iv) white is targeted but red is selected (incorrect response). For each experimental run, the average response and standard derivations are given and the number of times of occurrence of each outcome.

\begin{tabular}{|c|c|c|c|c|c|c|}
\hline & & $\begin{array}{l}\text { Red target } \\
\text { red } \\
\text { selected } \checkmark\end{array}$ & $\begin{array}{l}\text { Red target } \\
\text { white } \\
\text { selected } \times\end{array}$ & $\begin{array}{l}\text { White target } \\
\text { white } \\
\text { selected } \checkmark\end{array}$ & $\begin{array}{l}\text { White target } \\
\text { red } \\
\text { selected } \times\end{array}$ & Accuracy \\
\hline $\begin{array}{l}\text { Subject } 1 \\
\text { Exp. } 1\end{array}$ & $\begin{array}{l}\text { Avg. } \\
\text { St. Dev. } \\
\text { No. of trials }\end{array}$ & $\begin{array}{l}0.74 \\
0.36 \\
4\end{array}$ & $\begin{array}{l}- \\
\overline{0}\end{array}$ & $\begin{array}{l}0.40 \\
0.22 \\
5\end{array}$ & $\begin{array}{l}0.33 \\
- \\
1\end{array}$ & $90 \%$ \\
\hline Exp. 2 & $\begin{array}{l}\text { Avg. } \\
\text { St. Dev. } \\
\text { No. of trials }\end{array}$ & $\begin{array}{l}0.62 \\
0.50 \\
2\end{array}$ & $\begin{array}{l}0.39 \\
0.48 \\
2\end{array}$ & $\begin{array}{l}0.41 \\
0.19 \\
6\end{array}$ & $\begin{array}{l}- \\
\overline{0}\end{array}$ & $80 \%$ \\
\hline Exp. 3 & $\begin{array}{l}\text { Avg. } \\
\text { St. Dev. } \\
\text { No. of trials }\end{array}$ & $\begin{array}{l}0.41 \\
0.19 \\
2\end{array}$ & $\begin{array}{l}- \\
- \\
0\end{array}$ & $\begin{array}{l}0.52 \\
0.20 \\
6\end{array}$ & $\begin{array}{l}0.57 \\
0.27 \\
2\end{array}$ & $80 \%$ \\
\hline Exp. 4 & $\begin{array}{l}\text { Avg. } \\
\text { St. Dev. } \\
\text { No. of trials }\end{array}$ & $\begin{array}{l}0.24 \\
0.02 \\
2\end{array}$ & $\begin{array}{l}0.22 \\
0.13 \\
2\end{array}$ & $\begin{array}{l}0.34 \\
0.19 \\
5\end{array}$ & $\begin{array}{l}0.22 \\
- \\
1\end{array}$ & $70 \%$ \\
\hline Exp. 5 & $\begin{array}{l}\text { Avg. } \\
\text { St. Dev. } \\
\text { No. of trials }\end{array}$ & $\begin{array}{l}0.08 \\
0.04 \\
2\end{array}$ & $\begin{array}{l}0.22 \\
0.13 \\
2\end{array}$ & $\begin{array}{l}0.34 \\
0.19 \\
5\end{array}$ & $\begin{array}{l}0.22 \\
- \\
1\end{array}$ & $70 \%$ \\
\hline Exp. 6 & $\begin{array}{l}\text { Avg. } \\
\text { St. Dev. } \\
\text { No. of trials }\end{array}$ & $\begin{array}{l}0.79 \\
0.49 \\
4\end{array}$ & $\begin{array}{l}- \\
- \\
0\end{array}$ & $\begin{array}{l}0.22 \\
0.07 \\
5\end{array}$ & $\begin{array}{l}0.01 \\
- \\
1\end{array}$ & $80 \%$ \\
\hline $\begin{array}{l}\text { Subject } 2 \\
\text { Exp. } 1\end{array}$ & $\begin{array}{l}\text { Avg. } \\
\text { St. Dev. } \\
\text { No. of trials }\end{array}$ & $\begin{array}{l}0.47 \\
0.40 \\
3\end{array}$ & $\begin{array}{l}0.41 \\
- \\
1\end{array}$ & $\begin{array}{l}0.43 \\
0.29 \\
6\end{array}$ & $\begin{array}{l}- \\
\overline{0} \\
0\end{array}$ & $90 \%$ \\
\hline $\begin{array}{l}\text { Subject } 3 \\
\text { Exp. } 1\end{array}$ & $\begin{array}{l}\text { Avg. } \\
\text { St. Dev. } \\
\text { No. of trials }\end{array}$ & $\begin{array}{l}0.35 \\
- \\
1\end{array}$ & $\begin{array}{l}0.25 \\
0.12 \\
2\end{array}$ & $\begin{array}{l}0.16 \\
0.09 \\
6\end{array}$ & $\begin{array}{l}0.17 \\
- \\
1\end{array}$ & $70 \%$ \\
\hline Exp. 2 & $\begin{array}{l}\text { Avg. } \\
\text { St. Dev. } \\
\text { No. of trials }\end{array}$ & $\begin{array}{l}1.11 \\
0.24 \\
3\end{array}$ & $\begin{array}{l}- \\
\overline{0}\end{array}$ & $\begin{array}{l}0.66 \\
0.45 \\
6\end{array}$ & $\begin{array}{l}0.67 \\
- \\
1\end{array}$ & $90 \%$ \\
\hline
\end{tabular}

imagery. There is a significant increase in $\mathrm{HbO}$ concentrations when imagery is performed. Table 1 presents the average response of each experimental run for each subject. Subject performance is evaluated simply by the ratio of the number of correct classifications to the number of trials. Subjects were able to achieve reasonable accuracy levels, with an average accuracy of $80 \%$, and in the case of subject 1 performance was consistent throughout the week.

\section{Discussion}

The results as given are limited in what they can tell us about this particular instrument's competency as a general purpose BCI given the relatively limited testing. However, the results do demonstrate that this simple instrument allowed some degree of BCI operation with quite basic technology and additionally some insight was gained into general issues with such BCI paradigms.

The imagery tasks involved were straightforward concepts, although subjects reported the need to develop an individual strategy to perform mental imagery. This included the imagination of motor functions beyond hand movement, e.g. pedalling a bicycle with one leg, kicking footballs and sifting one's fingers though a bucket of grain. The use of continuous feedback as explored in pilot studies [3] may be useful for helping users to cultivate such strategies. Feedback provided by the end result, as given by 'Mindswitch', has proved sufficient in EEG BCI systems; however continual feedback such as using virtual reality has been shown to improve task performance [22]. The benefit of increasing feedback to emphasize or impede the motor imagery process needs to be examined further. During rest intervals, envisaging concepts completely unrelated to movement was found to be difficult because motion is associated with so many phenomena [23]. Therefore, it may help to integrate some graphical representations for this purpose.

The overall system performance may be improved through a number of methods, including signal augmentation, signal classification and noise reduction. Information content may be enhanced by augmenting the signal with $\mathrm{Hb}$ concentration changes. The Hb level is the target parameter in fMRI studies and should yield additional features. The system configuration needs to be optimized to improve the integrity of the smaller $\mathrm{Hb}$ signal change, which is susceptible to cross-talk effects due to assumptions made in the modified Beer-Lambert law 
algorithm. By choosing optimum wavelengths of light, the $\mathrm{Hb}$ signal integrity may be improved [24]. Addition of a third wavelength would also allow respiratory enzyme cytochrome oxidase concentration changes to be measured which are also linked with brain functional activity. This could then be used as an additional feature in event classification. An alternative approach to signal augmentation would be to develop a hybrid system by integrating other brain monitoring modalities, such as EEG, with the optimal deployment of NIR techniques. Optical signals are ideal for multi-modal studies, as the light signal does not interfere with electrical or magnetic fields.

Clearly, the switching process as demonstrated is slow as a result of the comparative approach based on a relative threshold. Some pilot studies [3] that we carried out used absolute thresholds, i.e. where classification of an event occurs if the change in the average $\mathrm{HbO}$ concentration exceeds a pre-defined reference level. This yielded faster speeds of operation, but at the cost of less flexibility and accuracy. Part of the reasoning behind the approach taken in Mindswitch was to facilitate greater flexibility, allowing easy accommodation of new users. A threshold does not need to be calculated for each user over numerous trials. Moreover, an ill-defined threshold or signal drift can lead to increased errors. However, in order to achieve faster information transfer rates, thresholding may be preferable. This may be combined with feature classification methods and asynchronous mode implementation. More sophisticated signal processing is required to prevent the occurrence of false positives due to physiological noise effects as we have discussed in a previous work [25]. Support vector machines and hidden Markov models have been demonstrated with off-line data [4], and show some promise.

Another factor affecting the signal integrity is the effect of superficial layers on the detected signal. These layers of tissue are assumed to have a constant attenuation effect on the light signal; however, there is a slight effect due to extracerebral signal components [26]. This can be minimized by using multiple source-detector arrangements with different interoptode distances, which would result in different penetration depths, thereby isolating the effect of superficial layers of tissue.

One of the major limitations of fNIRS is the nature of the haemodynamic response which limits the information transfer rate given that vascular changes occur over a number of seconds. Multiple channels and additional mental tasks may be used to overcome this issue. Further investigation is needed into the variety of mental tasks that can be used as control channels. A recent fMRI study has used four different tasks to provide 2D cursor control [27]. The mental tasks were left- and right-hand movement imageries, mental calculation and mental speech generation. Such mental processes have been detected using NIRS techniques and could therefore be utilized [19, 28, 29].

Recent studies have reported fast optical signals that may correlate with the event-related potentials detected by EEG. The optical counterpart of EP has been termed the eventrelated optical signal (EROS) [30]. A recent study revealed an EROS, showing a response of the order of milliseconds, correlating to the frequency of hand movement [31]. To achieve optimum speed, acquiring the fast optical signal will be essential. However, currently thousands of averages are required for its successful acquisition and the signal is highly localized. For EROS to be practical for BCI deployment, more robust and reliable methods of measurement must first be accomplished.

While physiological noise effects and motion artefacts obviously create errors, the occurrence of subject error must also be taken into consideration. Subjects reported that a lack of concentration or momentary confusion during the tasks led to erroneous target selections.

\section{Conclusions}

We have shown how a simplified fNIRS device designed to detect haemodynamic responses arising from mental imagery processes can be used as a control channel for a computer application. The resulting 'Mindswitch', in a synchronous mode, uses a single channel placed on a region giving a pronounced optical response. This work demonstrates the suitability of an optical interrogation modality to provide a control input signal to a BCI. The advantages of optical systems include safety, accessibility and non-invasiveness. The cognitive processes detectable with NIRS allow straightforward mental tasks to be used in controlling the optical BCI. The ultimate potential of fNIRS$\mathrm{BCI}$ is dependent on increasing throughput and accuracy to rates useful enough for their adaptation by their intended users. This may be done by integrating more control channels, by seeking a faster optical response correlated to neuronal activity $[30,31]$ or by the addition of complementary functional brain imaging modality. It is hoped that this work will foster further investigations into the application of NIRS signals for a new era of $\mathrm{BCI}$ development.

\section{Acknowledgments}

This work was carried out at Biomedical Engineering Research Group, National University of Ireland Maynooth, Maynooth, County Kildare, Ireland, and supported by Higher Education Authority/Media Lab Europe Collaboration Award-(HEA MMRP 2001 WARD).

\section{References}

[1] Wolpaw J, Birbaumer N, Heetderks W, Farland D, Peckham P, Schalk G, Donchin E, Quatrano L, Robinson C and Vaughan T 2000 Brain-computer interface technology: a review of the first international meeting IEEE Trans. Rehabil. Eng. 8 164-73

[2] Villringer A, Planck J, Hock C, Schleinkofer L and Dirnagl U 1993 Near infrared spectroscopy(NIRS): a new tool to study hemodynamic changes during activation of brain function in human adults Neurosci. Lett. 154 101-4

[3] Coyle S 2005 Near infrared spectroscopy for brain computer interfacing $P h D$ Thesis National University of Ireland, Maynooth

[4] Sitaram R, Zhang H, Guan C, Thulasidas M, Hoshi Y, Ishikawa A, Shimizu K and Birbaumer N 2006 Temporal classification of multichannel near-infrared spectroscopy signals of motor imagery for developing a brain-computer interface Neuroimage 34 1416-27 
[5] Pfurtscheller G, Neuper C, Guger C, Harkam W, Ramoser H, Schloegl A, Obermaier B and Pregenzer M 2000 Current trends in Graz brain.computer interface (BCI) research IEEE Trans. Rehabil. Eng. 8 216-9

[6] Wolpaw E W, Birbaumer N, McFarland D, Pfurtscheller G and Vaughan T 2002 Brain computer interfaces for communication and control Clin. Neurophysiol. $113767-91$

[7] Kuebler A, Kotchoubey B, Kaiser J, Wolpaw E W and Birbaumer N 2001 Brain-computer communication: unlocking the locked in Psychol. Bull. 127 358-75

[8] Vaughan T M et al 2003 Brain-computer interface technology: a review of the second international meeting IEEE Trans. Neural Syst. Rehabil. Eng. 11 94-109

[9] Weiskopf N, Veit R, Erb M, Mathiak K, Grodd W, Goebel R and Birbaumer N 2003 Physiological self-regulation of regional brain activity using real-time functional magnetic resonance imaging (fMRI): methodology and exemplary data Neuroimage 19 577-86

[10] Laitinen L 2003 Neuromagnetic sensorimotor signals in brain computer interfaces Master Thesis Helsinki University of Technology, Helsinki

[11] Coyle S, Ward T, Markham C and McDarby G 2004 On the suitability of near-infrared systems for next generation brain computer interfaces Physiol. Meas. 25 815-22

[12] Coyle S, Ward T and Markham C 2004 An optical brain computer interface Biomedizinische Technik Proc. 2nd Int. Brain-Computer Interface Workshop and Training Course vol 49 pp 45-6

[13] Rolfe P 2000 In Vivo Near-infrared spectroscopy Annu. Rev. Biomed. Eng. 2 715-54

[14] Gratton G, Fabiani M, Elbert T and Rockstroh B 2003 Seeing right through you: Applications of optical imaging to the study of the human brain Psychophysiology 40 487-91

[15] Cope M and Delpy D T 1988 A system for the long-term measurement of cerebral blood and tissue oxygenation in newborn infants by near infra-red transillumination $\mathrm{Med}$. Biol. Eng. Comput. 26 289-94

[16] Chance B and Villringer A 1997 Non-invasive optical spectroscopy and imaging of human brain function Trends Neurosci. 20 435-42

[17] Strangman G, Boas D and Sutton J P 2002 Non-invasive neuroimaging using near-infrared light Biol. Psychiat. 52 679-93

[18] Tachtsidis I, Elwell C E, Leung S L, Lee C, Smith M and Delpy D T 2004 Investigation of cerebral haemodynamics by near-infrared spectroscopy in young healthy volunteers reveals posture-dependent spontaneous oscillations Physiol. Meas. 25 437-45

[19] Coyle S, Ward T and Markham C 2003 Cerebral blood flow changes related to motor imagery, using near-infrared spectroscopy (NIRS) World Congress on Medical Physics and Biomedical Engineering (Sydney, Australia)

[20] Mason S G and Birch G E 2000 A Brain-controlled switch for asynchronous control applications IEEE Trans. Biomed. Eng. 47 1297-307

[21] Neuper C, Scherer R, Reiner M and Pfurtscheller G 2005 Imagery of motor actions: differential effects of kinesthetic and visual.motor mode of imagery in single-trial EEG Cogn. Brain Res. 25 668-77

[22] Leeb R et al 2006 Walking by thinking:the brainwaves are crucial, not the muscles Presence 15 500-14

[23] de Lafuente V and Romo R 2004 Language abilities of motor cortex Neuron 41 178-80

[24] Strangman G, Franceschini M A and Boas D A 2003 Factors affecting the accuracy of near-infrared spectroscopy concentration calculations for focal changes in oxygenation parameters Neuroimage 18 865-79

[25] Coyle S, Ward T and Markham C 2004 Physiological noise in near-infrared spectroscopy: implications for optical brain computer interfacing 26th Annual Int. Conf. of the IEEE Engineering in Medicine and Biology Society (San Francisco)

[26] Kohl-Bareis M, Obrig H, Steinbrink J, Uludag K and Villringer A 2002 Noninvasive monitoring of cerebral blood flow by a dye bolus method: separation of brain from skin and skull signals J. Biomed. Opt. 7 464-70

[27] Yoo S, Fairneny T, Chen N, Choo S, Panych L P, Park H, Lee $\mathrm{S}$ and Jolesz F A 2004 Brain-computer interface using fMRI: spatial navigation by thoughts Neuroreport 15 1591-5

[28] Hoshi Y and Tamura M 1993 Detection of dynamic changes in cerebral oxygenation coupled to neuronal function during mental work in man Neurosci. Lett. 150 5-8

[29] Herrmann M J, Ehlis A C and Fallgatter A J 2003 Frontal activation during a verbal fluency task as measured by near-infrared spectroscopy Brain Res. Bull. 61 51-6

[30] Gratton G and Fabiani M 2001 Shedding light on brain function: the event-related optical signal Trends $\operatorname{Cog}$. Sci. 5 357-63

[31] Wolf M, Wolf U, Choi J H, Gupta R, Safonova L P, Michalos A and Gratton E 2002 Functional frequency-domain near-infrared spectroscopy neuronal signal in the motor cortex Neuroimage 17 1868-75 